\title{
On the $\mathcal{N}=2$ superconformal index and eigenfunctions of the elliptic RS model
}

\author{
Shlomo S. Razamat \\ School of Natural Sciences, Institute for Advanced Study, Princeton, NJ 08540, USA
}

\begin{abstract}
We define an infinite sequence of superconformal indices, $\mathcal{I}_{n}$, generalizing the Schur index for $\mathcal{N}=2$ theories. For theories of class $\mathcal{S}$ we then suggest a recursive technique to completely determine $\mathcal{I}_{n}$. The information encoded in the sequence of indices is equivalent to the $\mathcal{N}=2$ superconformal index depending on a maximal set of fugacities. Mathematically, the procedure suggested in this note provides a perturbative algorithm for computing a set of eigenfunctions of the elliptic Ruijsenaars-Schneider model.
\end{abstract}

August 2013 


\section{Introduction}

It often happens that some of the properties of a given physical system can be constrained by exploiting the symmetries, and other robust properties, of that system. In more rare circumstances some of the observables are completely determined by constraints imposed by robust considerations. Such observables are especially useful if, say due to strong coupling, direct computations in the theory describing the physical system are not feasible.

An example of a quantity fixed by symmetries and robust properties is the superconformal index of the $\mathcal{N}=2$ quantum field theories of class $\mathcal{S}$ [1,2]. The superconformal index is a twisted supersymmetric partition function on $\mathbb{S}^{3} \times \mathbb{S}^{1}$ and thus is severely constrained by supersymmetry. Moreover, due to an intricate web of relations between different theories of class $\mathcal{S}$, S-dualities and RG flows, under certain mathematical assumptions to be discussed below, the physical task of determining the superconformal index can be reduced to a well defined mathematical problem of diagonalizing a set of commuting difference operators [3]. The purpose of this brief note is to argue that this mathematical problem itself can be solved by exploiting further the S-dualities relating the theories of class $\mathcal{S}$.

The difference operators one has to diagonalize in order to determine the superconformal index are the elliptic Ruijsenaars-Schneider (RS) Hamiltonians $[4,5,6]$. We will see that S-duality properties relating different theories of class $\mathcal{S}$ of type $A_{N-1}$ can be translated to a system of coupled integral equations for the eigenfunctions of elliptic RS Hamiltonians (and additional un-known functions). A solution of this system of equations is known in closed form in particular limits of the parameters. We will then construct the eigenfunctions for the general case by perturbing around one such limit, the so called Schur index $[7,8]$.

The Schur index has several interesting properties: e.g., it is related to qYM in $2 \mathrm{~d}[7,9,10,11]$, it has interesting modular properties and is given in terms of q-polyGamma functions [12]. This version of the index depends only on one fugacity, which we denote by $q$, coupled to charges of the superconformal symmetry group. This is to be contrasted with the most general $4 \mathrm{~d} \mathcal{N}=2$ index one can define depending on three superconformal fugacities [13], usually denoted $(p, q, t)$ : the Schur index is obtained by setting $t=q$. We will define the general $\mathcal{N}=2$ index as a power series in $T=(t / q)^{\frac{1}{2}}-1$. The coefficients of this series are indices by themselves, which we will denote by $\mathcal{I}_{n}$. We then solve for the eigenfunctions of elliptic RS as Taylor series in $T$. Knowing these eigenfunctions up to order $\widetilde{n}$ in $T$ is sufficient to determine the indices $\mathcal{I}_{n \leq \widetilde{n}}$. 
Let us mention here that the interplay between quantum mechanical integrable models and gauge theories in higher dimensions has a rich history. For example, an explicit relation between the trigonometric RS model and gauged $G / G$ WZW model was discussed in [14]. In [15] a generalization of this relation to elliptic non-relativistic models appeared, while the elliptic RS model was found to be connected to 5 d gauge theories on a circle [16]. For a nice review of these, and other, relations and more references one can consult [17]. More recently, the quantum elliptic $\mathrm{RS}$ model made its appearance in [18] while considering $\mathcal{N}=2$ theories in the $\Omega$-background. ${ }^{1}$

This note is organized as follows. We start in section 1 with a definition of a sequence of $\mathcal{N}=2$ indices, $\mathcal{I}_{n}$, generalizing the Schur index. In section 2 we discuss $A_{1}$ theories of class $\mathcal{S}$ and spell out a simple algorithm to determine a set of eigenfunctions of the elliptic RS model, which are needed to compute $\mathcal{I}_{n}$. In section 3, as a concrete example of analysis for $A_{N-1}$ theories of class $\mathcal{S}$ with $N>2$, we extend the algorithm to the $A_{2}$ case. Finally, in section 4 we briefly comment on our results.

Before proceeding to the main part of the note let us set several mathematical notations. The elliptic Gamma function is defined as,

$$
\Gamma(x ; p, q)=\prod_{i, j \geq 0} \frac{1-\frac{p q}{x} p^{i} q^{j}}{1-x p^{i} q^{j}} .
$$

The q-Pochhammer symbol and the theta-function are given by,

$$
(x ; q)=\prod_{i \geq 0}\left(1-x q^{i}\right), \quad \theta(x ; q)=(x ; q)\left(\frac{q}{x} ; q\right),
$$

and we will find it useful to define the following

$$
\kappa_{n}=\frac{\left((q ; q)(p ; p) \Gamma\left(\frac{p q}{t} ; p, q\right)\right)^{n}}{(n+1) !} .
$$

Finally, we use the usual short-hand notation,

$$
f\left(z^{ \pm 1} y^{ \pm 1} \cdots\right)=f(z y \cdots) f\left(z^{-1} y \cdots\right) f\left(z y^{-1} \cdots\right) f\left(z^{-1} y^{-1} \cdots\right) .
$$

1 It would be interesting to work out in detail the relations between these appearances of RS models in $\mathcal{N}=2$ gauge theories and the index: see e.g., [19] for a suggestion. 


\section{A sequence of indices}

The $\mathcal{N}=2$ superconformal index is given by the following trace formula,

$$
\mathcal{I}\left(p, q, t,\left\{a_{\ell}\right\}\right)=\operatorname{Tr}(-1)^{F} p^{j_{2}+j_{1}-r} q^{j_{2}-j_{1}-r} t^{r+R} e^{-\beta \delta} \prod_{\ell} a_{\ell}^{f_{\ell}}
$$

The trace is over the states of a $4 d \mathcal{N}=2$ superconformal theory in radial quantization. Here $F$ is the fermion number, $\left(j_{1}, j_{2}\right)$ the Cartans of the rotation group $S U(2)_{1} \times S U(2)_{2} \sim$ $S O(4), R$ is the Cartan of $S U(2)_{R}$ R-symmetry, $r$ the generator of $U(1)_{r}$ R-symmetry, and $f_{\ell}$ are Cartans of the flavor symmetry (if there is any). The charge $\delta$ is an anti-commutator of two supercharges, ${ }^{2}$

$$
\delta=2\left\{\widetilde{\mathcal{Q}}_{1, \dot{-}},\left(\widetilde{\mathcal{Q}}_{1, \dot{-}}\right)^{\dagger}\right\}=E-2 j_{2}-2 R+r .
$$

All the charges in $(2.1)$ commute with $\widetilde{\mathcal{Q}}_{1,-}$ and thus the index is independnet of $\delta$ : we will omit it from the discussion in what follows. The supercharge $\widetilde{\mathcal{Q}}_{1,-}$. has the following charges $\left(R, r, j_{1}, j_{2}\right)=\left(\frac{1}{2},-\frac{1}{2}, 0,-\frac{1}{2}\right)$.

We introduce a new parameter $T$,

$$
1+T=(t / q)^{1 / 2}
$$

and define a sequence of indices, $\mathcal{I}_{n}$, by the following

$$
\mathcal{I}=\sum_{n=0}^{\infty} T^{n} \mathcal{I}_{n}
$$

In all cases where the index can be directly computed such an expansion in $T$ is convergent and we assume this to be true for all theories we will discuss. The indices $\mathcal{I}_{n}$ have a simple physical meaning

$$
\mathcal{I}_{n}=\operatorname{Tr}(-1)^{F} P_{n}(r+R) p^{j_{2}+j_{1}-r} q^{j_{2}-j_{1}+R} \prod_{\ell} a_{\ell}^{f_{\ell}},
$$

with $P_{0}(z)=1$ and $P_{n}(z)=\frac{1}{n !} \prod_{\ell=0}^{n-1}(2 z-\ell)$. The index $\mathcal{I}_{0}$ is the Schur index $[7,8]$,

2 We use the notations of [8]. 


$$
\mathcal{I}_{0}=\operatorname{Tr}(-1)^{F} p^{j_{2}+j_{1}-r} q^{j_{2}-j_{1}+R} \prod_{\ell} a_{\ell}^{f_{\ell}}=\operatorname{Tr}(-1)^{F} q^{j_{2}-j_{1}+R} \prod_{\ell} a_{\ell}^{f_{\ell}}
$$

Here the dependence on $p$ completely drops out since the charge it couples to is (up to admixture of $\delta$ ) an anticommutator of a supercharge, $\mathcal{Q}_{1,+}$ (with charges $\left(R, r, j_{1}, j_{2}\right)=$ $\left.\left(\frac{1}{2}, \frac{1}{2}, \frac{1}{2}, 0\right)\right)$, which commutes with all the other charges appearing in the index. The indices $\mathcal{I}_{n>0}$, however, do depend on $p$ since $\mathcal{Q}_{1,+}$ has non vanishing $r+R$ charge, e.g.

$$
\mathcal{I}_{1}=\operatorname{Tr}(-1)^{F} 2(r+R) p^{j_{2}+j_{1}-r} q^{j_{2}-j_{1}+R} \prod_{\ell} a_{\ell}^{f_{\ell}}
$$

The coefficients of the term $p^{n} q^{m} \prod_{\ell} a_{\ell}^{f_{\ell}}$ in expansion of $\mathcal{I}_{1}$ give a graded sum over Rcharges of states with $j_{2}+j_{1}-r=n, j_{2}-j_{1}+R=m, \delta=0$, and the flavor charges $f_{\ell}$. Note that the indices $\mathcal{I}_{n}$ do not have the symmmetry exchanging $p$ and $q$.

Using the algorithm of this note one can compute the indices $\mathcal{I}_{n}$ for theories of class $\mathcal{S}$. We will give explicit ingredients for the computation of $\mathcal{I}_{1}$ for $A_{1}$ quivers and $A_{2}$ quivers.

\section{The intergral equation: $A_{1}$ case}

Theories of class $\mathcal{S}$ of type $A_{N-1}$ are obtained by compactifying $M 5$-branes on a punctured Riemann surface $[1,2]$. These are $4 d \mathcal{N}=2$ theories labeled by the choice of the punctured Riemann surface. The modular parameters of the Riemann surface correspond to exactly marginal couplings of the $4 d$ theory. The superconformal index is independent of such continuous parameters of the theory and thus can be thought of as a topological invariant associated to the underlying Riemann surface [20]. The types of punctures are classified by partitions of $N$ and encode information about the flavor symmetry of the $4 d$ theory.

In this section we discuss the simplest case of theories of class $\mathcal{S}$, the $A_{1}$ generalized quivers. For $A_{1}$ case there is only one type of non-trivial puncture corresponding to an $S U(2)$ flavor symmetry. The index of a class $\mathcal{S}$ theory of $A_{1}$ type corresponding to genus $g$ Riemann surface with $s$ punctures ${ }^{3}$ can be constructed as follows. The index of a theory corresponding to three-punctured sphere (trinion) is

$$
\mathcal{I}^{(0,3)}(a, b, c)=\Gamma\left(t^{1 / 2} a^{ \pm 1} b^{ \pm 1} c^{ \pm 1} ; p, q\right) .
$$

3 We only discuss regular punctures in this note [21]. 
Here the parameters $a, b$, and $c$ are fugacities for the Cartans of the three $S U(2)$ flavor symmetries associated to the three punctures. The corresponding $4 d$ theory is a free hypermultiplet in the bi-fundamental representation of $S U(2) \times S U(2)$. Given two general Riemann surfaces and the corresponding indices one constructs the index of the theory associated to the Riemann surface obtained by gluing the two surfaces along a puncture,

$$
\begin{aligned}
& \mathcal{I}^{\left(g+g^{\prime}, s+s^{\prime}-2\right)}\left(\left\{a_{i}\right\}_{i=1}^{s+s^{\prime}-1}\right)= \\
& \quad \kappa_{1} \oint \frac{d z}{2 \pi i z} \frac{\Gamma\left(\frac{p q}{t} z^{ \pm 2} ; p, q\right)}{\Gamma\left(z^{ \pm 2} ; p, q\right)} \mathcal{I}^{(g, s)}\left(z,\left\{a_{i}\right\}_{i=1}^{s-1}\right) \mathcal{I}^{\left(g^{\prime}, s^{\prime}\right)}\left(z^{-1},\left\{a_{i}\right\}_{i=s}^{s+s^{\prime}-2}\right) .
\end{aligned}
$$

Gluing two Riemann surfaces corresponds to gauging the diagonal combination of the $S U(2)$ flavor symmetries associated to the glued punctures. The ratio of two elliptic Gamma functions and $\kappa_{1}$ in (3.2) come from the index of the $\mathcal{N}=2$ vector multiplet (and the Haar measure) one introduces when a symmetry is gauged. The index for any Riemann surface is constructed recursively by decomposing the surface into three-punctured spheres (pairs-of-pants) and gluing them together.

Our perturbative procedure to determine the indices $\mathcal{I}_{n}$ is based on two main assumptions: one physical and one mathematical. The physics we will assume to be correct is that the theories of class $\mathcal{S}$ enjoy the action of S-duality as discussed in [1]. In particular this means that the indices computed in any duality frame are equal [20]: this statement is a mathematically proven fact at the level of the index for the $A_{1}$ theories of class $\mathcal{S}$ (with regular punctures) [22]. Saying it differently, S-duality is the assumption that the recursive procedure defined above to construct indices of theories associated to general Riemann surfaces is well defined, i.e., independent of the pairs-of-pants decomposition.

The main mathematical assumption we will make is that the index of a theory of class $\mathcal{S}$ can be written in the following form, ${ }^{4}$

$$
\mathcal{I}^{(g, s)}\left(p, q, t ;\left\{a_{\ell}\right\}\right)=\sum_{\lambda=0}^{\infty}\left[\mathcal{C}_{\lambda}\right]^{2 g-2+s} \prod_{i=1}^{s} \psi_{\lambda}\left(a_{i} ; p, q, t\right)
$$

4 Here we assume that for $g=0$ the number of punctures is bigger than two, and for $g=1$ there is at least one puncture. 
where the functions $\psi_{\lambda}(a ; p, q, t)$ form an orthonormal set of functions under the vector multiplet measure, ${ }^{5}$

$$
\left\langle\left\langle\psi_{\lambda}, \psi_{\mu}\right\rangle\right\rangle=\kappa_{1} \oint \frac{d z}{2 \pi i z} \frac{\Gamma\left(\frac{p q}{t} z^{ \pm 2} ; p, q\right)}{\Gamma\left(z^{ \pm 2} ; p, q\right)} \psi_{\lambda}(z) \psi_{\mu}(z)=\delta_{\lambda, \mu} .
$$

One can use the physical assumption above to motivate the expression (3.3) [8]. Take the index of the $A_{1}$ trinion (3.1) and expand it in some convenient complete set of functions, $f_{\mu}(z)$, orthonormal under the vector multiplet measure (3.4). Such an expansion defines a set of symmetric structure constants, $C_{\mu \nu \lambda}$. These structure constants have to form an associative algebra following from the S-duality properties of the underlying theories. Then pending certain natural assumptions and convergence issues one can change a basis of the orthogonal functions such that the structure constants will be "diagonalized" to Frobenius form (3.3). In such a "diagonal" form the S-duality properties of the indices of $A_{1}$ theories are manifest. At least when one specializes the parameters $(p, q, t)$ to certain values there is a lot of evidence that the mathematical assumption (3.3) is correct. For example, when one specializes the fugacities to $p=0$ ( or $q=0$ ) the functions $\psi_{\lambda}(a)$ can be explicitly found to be related to Macdoanld polynomials [8], and taking $t=q$ these are related to Schur polynomials as we will discuss in the next sub-section. If the functions $\psi_{\lambda}(a)$ are known, utilizing (3.3) to compute the indices of general $A_{1}$ quivers is much simpler than applying the recursive procedure using (3.2). We thus turn to deriving our basic integral equation determining the eigenfunctions $\psi_{\lambda}(a)$.

It will be convenient for us to absorb a factor of $\mathcal{C}_{\lambda}$ into the definition of the functions,

$$
\hat{\psi}_{\lambda}(z ; p, q, t)=\mathcal{C}_{\lambda} \psi_{\lambda}(z ; p, q, t)
$$

The basic example of an index, the index of the theory corresponding to the threepunctured sphere $(3.1)$, can be then written as

$$
\mathcal{I}^{(0,3)}=\Gamma\left(t^{1 / 2} a^{ \pm 1} b^{ \pm 1} c^{ \pm 1} ; p, q\right)=\sum_{\lambda=0}^{\infty} \mathcal{C}_{\lambda}^{-2} \hat{\psi}_{\lambda}(a ; p, q, t) \hat{\psi}_{\lambda}(b ; p, q, t) \hat{\psi}_{\lambda}(c ; p, q, t) .
$$

Using the orthogonality property (3.4) we immediately arrive at our basic integral equation for $\hat{\psi}_{\lambda}(z ; p, q, t),{ }^{6}$

5 We will often omit the parameters $(p, q, t)$ for the sake of brevity.

6 This is an elliptic generalization of the product formula for $A_{1}$ Macdonald polynomials [23]. 


$$
\begin{aligned}
& \hat{\psi}_{\lambda}(y ; p, q, t) \hat{\psi}_{\lambda}(a ; p, q, t)= \\
& \quad \kappa_{1} \oint \frac{d z}{2 \pi i z} \frac{\Gamma\left(\frac{p q}{t} z^{ \pm 2} ; p, q\right)}{\Gamma\left(z^{ \pm 2} ; p, q\right)} \hat{\psi}_{\lambda}(z ; p, q, t) \Gamma\left(t^{1 / 2} z^{ \pm 1} y^{ \pm 1} a^{ \pm 1} ; p, q\right) .
\end{aligned}
$$

In the next sub-section we will argue that given the particular solution of this equation for $t=q$, it uniquely fixes $\hat{\psi}_{\lambda}(z ; p, q, t)$ for $t \neq q$.

Let us now comment on the relation of the above to the elliptic Ruijsenaars-Schneider model. It has been argued in [3] that there is a set of difference operators, $\mathfrak{S}_{(m, n)}^{(p, q, t)}$, for which the indices $\mathcal{I}^{(g, s)}$ are kernel functions (see e.g. $[24,25,26,27]$ for discussion of kernel functions $){ }^{7}$

$$
\mathfrak{S}_{(m, n)}^{(p, q, t)}\left(a_{\alpha}\right) \cdot \mathcal{I}^{(g, s)}\left(\left\{a_{i}\right\}\right)=\mathfrak{S}_{(m, n)}^{(p, q, t)}\left(a_{\beta}\right) \cdot \mathcal{I}^{(g, s)}\left(\left\{a_{i}\right\}\right), \quad \forall \alpha, \beta \in\{1,2, \cdots s\}
$$

These operators can be found in [3] and here we mention explicitly only the most basic one,

$$
\mathfrak{S}_{(1,0)}^{(p, q, t)}\left(\left\{z_{\ell}\right\}\right) \cdot f\left(\left\{z_{\ell}\right\}\right)=\sum_{i=1}^{N} \prod_{j \neq i} \frac{\theta\left(\frac{t}{p} z_{i} / z_{j} ; q\right)}{\theta\left(z_{j} / z_{i} ; q\right)} f\left(\left\{z_{\ell} \rightarrow p^{\frac{1}{N}-\delta_{\ell i}} z_{\ell}\right\}\right)
$$

We wrote this operator for $A_{N-1}$ case, so for $A_{1}$ one just has to set $N=2$ in the above. The difference operators $\mathfrak{S}_{(0,1)}^{(p, q, t)}$ is obtained by exchanging $p$ and $q, \mathfrak{S}_{(0,1)}^{(p, q, t)}=\mathfrak{S}_{(1,0)}^{(q, p, t)}$. The operator (3.9) is, up to conjugation with a simple function [3], the basic Hamiltonian of the elliptic Ruijsenaars-Schneider model. The operators (3.9) are self-adjoint under the natural measure in our problem, eq. (3.4).

The property (3.8) together with the assumption (3.3) and the orthogonality (3.4) imply that the functions $\hat{\psi}_{\lambda}(z ; p, q, t)$ are eigenfunctions of (3.9) and its generalizations

7 Kernel functions were instrumental in the perturbative solution of the non-relativistic elliptic Calogero-Moser model [24,28]. 
appearing in [3]. For example take $\mathcal{I}^{(1,3)}$ and act on it with $(3.9)$,

$$
\begin{aligned}
& \sum_{\lambda=0}^{\infty}\left[\mathfrak{S}_{(1,0)}^{(p, q, t)}\left(a_{1}\right) \hat{\psi}_{\lambda}\left(a_{1} ; p, q, t\right)\right] \prod_{i=2,3} \hat{\psi}_{\lambda}\left(a_{i} ; p, q, t\right)= \\
& \sum_{\lambda=0}^{\infty}\left[\mathfrak{S}_{(1,0)}^{(p, q, t)}\left(a_{2}\right) \hat{\psi}_{\lambda}\left(a_{2} ; p, q, t\right)\right] \prod_{i=1,3} \hat{\psi}_{\lambda}\left(a_{i} ; p, q, t\right), \\
& \frac{\downarrow}{\mathfrak{S}_{(1,0)}^{(p, q, t)}\left(a_{1}\right) \hat{\psi}_{\lambda}\left(a_{1} ; p, q, t\right)} \\
& \hat{\psi}_{\lambda}\left(a_{1} ; p, q, t\right)
\end{aligned}
$$

Going from first to second equality we applied orthogonality of the eigenfunctions $\hat{\psi}_{\lambda}\left(a_{3} ; p, q, t\right)$. Here we have also assumed that the order of performing the infinite sum and acting with the difference operator is immaterial. In degeneration limits, say $p=0$ or $t=q$, the functions $\hat{\psi}_{\lambda}(z ; p, q, t)$ can be explicitly shown to be eigenfunctions of (3.9). In what follows we will give some evidence that indeed this is the case beyond $t=q$, and in particular that our mathematical and physical assumptions are consistent.

\subsection{Recursive solution}

Let us now turn to solving the integral equations (3.7) perturbatively starting with a known solution for specialized parameters, $t=q$. We set the small parameter $T$ to be as in $(2.3)$,

$$
1+T=(t / q)^{1 / 2}
$$

We define

$$
\Delta(z, y, a ; p, q, t)=\kappa_{1} \frac{\Gamma\left(\frac{p q}{t} z^{ \pm 2} ; p, q\right)}{\Gamma\left(z^{ \pm 2} ; p, q\right)} \Gamma\left(t^{1 / 2} z^{ \pm 1} y^{ \pm 1} a^{ \pm 1} ; p, q\right)=\sum_{n=0}^{\infty} T^{n} \Delta^{(n)}(z, y, a ; p, q)
$$

and

$$
\hat{\psi}_{\lambda}(z ; p, q, t)=\sum_{n=0}^{\infty} T^{n} \hat{\psi}_{\lambda}^{(n)}(z ; p, q)=\sum_{n=0}^{\infty} T^{n} \sum_{\mu=0}^{\infty} C_{\mu ; \lambda}^{(n)}(p, q) \hat{\psi}_{\mu}^{(0)}(z ; q)
$$

Here $\hat{\psi}_{\mu}^{(0)}(z ; q)$ is the solution to $(3.7)$ in the Schur case, $t=q$, which is known $[7,8]$, 


$$
\hat{\psi}_{\mu}^{(0)}(z ; q)=\frac{1}{1-q}\left(\prod_{\ell=0}^{\infty} \frac{1}{1-q^{1+\ell} z^{ \pm 2}}\right) \frac{\chi_{\mu}(z)}{\chi_{\mu}\left(q^{\frac{1}{2}}\right)}, \quad \chi_{\mu}(z)=\frac{z^{\mu+1}-z^{-\mu-1}}{z-z^{-1}}
$$

These functions are eigenfunctions of difference operators (3.9) when one takes $t=q$,

$$
\mathfrak{S}_{(1,0)}^{(p, q, q)} \cdot \hat{\psi}_{\mu}^{(0)}=\left(p^{-\frac{\mu}{2}}+p^{\frac{\mu}{2}+1}\right) \hat{\psi}_{\mu}^{(0)}, \quad \mathfrak{S}_{(0,1)}^{(p, q, q)} \cdot \hat{\psi}_{\mu}^{(0)}=\left(q^{-\frac{\mu}{2}}+q^{\frac{\mu}{2}+1}\right) \hat{\psi}_{\mu}^{(0)}
$$

The coefficients $C_{\mu ; \lambda}^{(n)}(q, p)$ are given by

$$
C_{\mu ; \lambda}^{(n)}(p, q)=\frac{\left\langle\hat{\psi}_{\lambda}^{(n)}(z ; p, q), \hat{\psi}_{\mu}^{(0)}(z ; q)\right\rangle}{\left\langle\hat{\psi}_{\mu}^{(0)}(z ; q), \hat{\psi}_{\mu}^{(0)}(z ; q)\right\rangle}
$$

with the inner product defined using the specialization of the vector multiplet measure (3.4) to the Schur case,

$$
<f, g>=\oint \frac{d z}{2 \pi i z} \Delta(z ; q) f(z) g(z), \quad \Delta(z ; q)=\frac{(q ; q)^{2}}{2} \theta\left(q z^{2} ; q\right) \theta\left(q z^{-2} ; q\right) .
$$

Using these definitions the integral equation (3.7) becomes an equation for $C_{\nu ; \lambda}^{(n)}$. Taking $n>0$ one obtains

$$
C_{\nu ; \lambda}^{(n)} \delta_{\mu}^{\lambda}+C_{\mu ; \lambda}^{(n)} \delta_{\nu}^{\lambda}+\sum_{k+l=n, k, l<n} C_{\nu ; \lambda}^{(k)} C_{\mu ; \lambda}^{(l)}=C_{\mu ; \lambda}^{(n)} \delta_{\mu \nu}+\sum_{\rho=0}^{\infty} \sum_{k+l=n, l<n} \Gamma_{\mu \nu}^{(k)}{ }_{\mu \nu}^{\rho} C_{\rho ; \lambda}^{(l)}
$$

where,

$$
\begin{aligned}
& \Gamma_{\mu \nu}^{(k)^{\rho}}= \\
& \oint \frac{d z}{2 \pi i z} \oint \frac{d y}{2 \pi i y} \oint \frac{d a}{2 \pi i a} \frac{\Delta^{(k)}(z, y, a ; p, q) \Delta(y ; q) \Delta(a ; q) \hat{\psi}_{\rho}^{(0)}(z ; q) \hat{\psi}_{\mu}^{(0)}(y ; q) \hat{\psi}_{\nu}^{(0)}(a ; q)}{\left\langle\hat{\psi}_{\mu}^{(0)}, \hat{\psi}_{\mu}^{(0)}\right\rangle\left\langle\hat{\psi}_{\nu}^{(0)}, \hat{\psi}_{\nu}^{(0)}\right\rangle}
\end{aligned}
$$

with $\Delta(z ; q)$, defined in (3.16), being the measure under which the functions (3.13) are orthogonal.

One can now recursively solve these equations. Taking $n=1$ we obtain immediately

$$
\begin{aligned}
& C_{\mu ; \lambda}^{(1)}=\Gamma_{\mu \lambda}^{(1)^{\lambda}}=-\Gamma_{\mu \mu}^{(1)^{\lambda}} \quad(\lambda \neq \mu), \quad \Gamma_{\mu \nu}^{(1)^{\rho}}=0 \quad(\rho \neq \mu, \rho \neq \nu, \mu \neq \nu), \\
& C_{\mu ; \mu}^{(1)}=\Gamma_{\mu \mu}^{(1)^{\mu}} .
\end{aligned}
$$


Note that the set of equations is overdetrmined and there are thus consistency conditions to be satisfied: for the procedure to have a solution the functions $\Gamma^{(1)}{ }_{\mu \nu}^{\rho}$ are not arbitrary. Similarly, at the second level, $n=2$, one obtains

$$
\begin{aligned}
& C_{\mu ; \lambda}^{(2)}=C_{\lambda ; \mu}^{(1)} C_{\mu ; \lambda}^{(1)}+\Gamma_{\mu \lambda}^{(2)^{\lambda}}=\left(C_{\mu ; \lambda}^{(1)}\right)^{2}+\sum_{\rho=0}^{\infty}(-1)^{\delta_{\mu \rho}} C_{\mu ; \rho}^{(1)} C_{\rho ; \lambda}^{(1)}-\Gamma_{\mu \mu}^{(2)^{\lambda}} \quad(\lambda \neq \mu), \\
& \Gamma_{\mu \nu}^{(2)}=C_{\mu ; \rho}^{(1)} C_{\nu ; \rho}^{(1)}-C_{\mu ; \rho}^{(1)} C_{\nu ; \mu}^{(1)}-C_{\nu ; \rho}^{(1)} C_{\mu ; \nu}^{(1)} \quad(\rho \neq \mu, \rho \neq \nu, \mu \neq \nu), \\
& C_{\mu ; \mu}^{(2)}=\Gamma_{\mu \mu}^{(2)^{\mu}}-\sum_{\rho=0, \rho \neq \mu}^{\infty} C_{\mu ; \rho}^{(1)} C_{\rho ; \mu}^{(1)} .
\end{aligned}
$$

And so on. As an exercise one can set $p=0$ and verify that the recursive procedure generates the known solutions [8],

$$
\hat{\psi}_{\mu}(z ; p=0, q, t)=\frac{\left(t^{2} ; q\right)}{(t ; q)}\left(\prod_{\ell=0}^{\infty} \frac{1}{1-t q^{\ell} z^{ \pm 2}}\right) \frac{P_{\mu}(z ; q, t)}{P_{\mu}\left(t^{\frac{1}{2}} ; q, t\right)}
$$

where $P_{\mu}(z ; q, t)$ are the Macdonald polynomials.

Our goal was to generate the solution for the elliptic case, and here are several eigenfunctions up to leading correction in $T$, and expanded to several orders in $p$ and $q,{ }^{8}$

$$
\begin{aligned}
& \hat{\psi}_{0}(z)= 1+q+2 q^{2}+T\left(2 q-2 p^{2}+4 p q+2 p^{2} q+6 q^{2}+8 p q^{2}+8 p^{2} q^{2}\right)+ \\
&\left(z^{2}+z^{-2}\right)\left(q+2 q^{2}+T\left(2 q+2 p q+2 p^{2} q+6 q^{2}+6 p q^{2}+6 p^{2} q^{2}\right)\right)+ \\
&\left(z^{4}+z^{-4}\right)\left(q^{2}+T\left(4 q^{2}+2 p q^{2}+4 p^{2} q^{2}\right)\right)+\cdots \\
& \hat{\psi}_{1}(z)=\left(z+z^{-1}\right)\left(q^{1 / 2}+q^{3 / 2}+T\left(q^{1 / 2}+q^{3 / 2}\left(3+4 p+4 p^{2}\right)\right)\right)+ \\
& \quad\left(z^{3}+z^{-3}\right)\left(q^{3 / 2}\left(1+T\left(3+2 p+2 p^{2}\right)\right)\right)+\cdots, \\
& \hat{\psi}_{2}(z)= q+2 q^{2}+T\left(2 q+2 p q+6 q^{2}+4 p q^{2}+4 p^{2} q^{2}\right)+ \\
&\left(z^{2}+z^{-2}\right)\left(q+q^{2}+T\left(2 q+4 q^{2}+4 p q^{2}+4 p^{2} q^{2}\right)\right)+ \\
&\left(z^{4}+z^{-4}\right)\left(q^{2}+T\left(4 q^{2}+2 p q^{2}+2 p^{2} q^{2}\right)\right)+\cdots, \\
& \hat{\psi}_{3}(z)=\left(z+z^{-1}\right) q^{3 / 2}\left(1+T\left(3+2 p+2 p^{2}\right)\right)+\left(z^{3}+z^{-3}\right) q^{3 / 2}(1+3 T)+\cdots .
\end{aligned}
$$

One can check that these eigenfunctions are orthogonal under the vector multiplet measure (3.4) and compute the eigenvalues. For example the eigenvalues of the $\lambda=0,1$ eigenfunctions with respect to the operator $\mathfrak{S}_{(1,0)}^{(p, q, t)}$ are

8 We performed explicit computations using Mathematica. 


$$
\begin{aligned}
& \mathcal{E}_{(1,0)}^{\lambda=0}=1+p+2 T\left(q+q^{2}-p-p q-p q^{2}+\ldots\right) \\
& \mathcal{E}_{(1,0)}^{\lambda=1}=p^{-\frac{1}{2}}+p^{\frac{3}{2}}+2 T\left(p^{-\frac{1}{2}} q^{2}-p^{\frac{3}{2}}\right)+\cdots
\end{aligned}
$$

From the general structure of the difference operator (3.9) it is possible to deduce that the (orthonormal) eigenfunctions have to satisfy [3]

$$
\psi_{\lambda}(z ; p, q, t)=\Gamma(t ; p, q) \Gamma\left(t z^{2} ; p, q\right) \Gamma\left(t z^{-2} ; p, q\right) \psi_{\lambda}\left(z ; p, q, \frac{p q}{t}\right)
$$

As yet another check of consistency of our procedure we verified, perturbatively in $p$ and $q$, that the functions (3.22) indeed satisfy this property. The norm of the eigenfunctions $\hat{\psi}_{\lambda}(z)$ with respect to the vector multiplet measure $(3.4)$ gives the structure constants $\mathcal{C}_{\lambda}$. Namely,

$$
\mathcal{C}_{\lambda}=\left(\left\langle\left\langle\hat{\psi}_{\lambda}, \hat{\psi}_{\lambda}\right\rangle\right\rangle\right)^{\frac{1}{2}}
$$

The norms of the lowest eigenfunctions are given by,

$$
\begin{aligned}
& \left\langle\left\langle\hat{\psi}_{0}, \hat{\psi}_{0}\right\rangle\right\rangle=1-2 q^{2}+\left(4 p q-6 q^{2}+p^{2}\left(-6+4 q^{2}\right)\right) T+\ldots \\
& \left\langle\left\langle\hat{\psi}_{1}, \hat{\psi}_{1}\right\rangle\right\rangle=q-2 q^{2}+\left(2 q-6 q^{2}+p\left(-2 q+4 q^{2}\right)+p^{2}\left(-2 q+4 q^{2}\right)\right) T+\ldots \\
& \left\langle\left\langle\hat{\psi}_{2}, \hat{\psi}_{2}\right\rangle\right\rangle=q^{2}+\left(4 q^{2}-2 p q^{2}-4 p^{2} q^{2}\right) T+\ldots
\end{aligned}
$$

From here it is immediate to compute the $\mathcal{I}_{1}$ index for any $A_{1}$ quiver. For example, this index for torus with one puncture is given by

$$
\mathcal{I}_{1}^{(1,1)}(a)=\sum_{\lambda=0}^{\infty} \hat{\psi}_{\lambda}^{(1)}(a ; p, q)=\left(\frac{1}{a}+a\right) q^{1 / 2}+4\left(1+\frac{1}{a^{2}}+a^{2}\right) q+2\left(3+\frac{1}{a^{2}}+a^{2}\right) p q+\cdots
$$

The same index can be computed by gluing together two legs of the trinion (3.1)

$$
\mathcal{I}_{1}^{(1,1)}(a)=\left[\frac{d}{d T} \kappa_{1} \oint \frac{d z}{2 \pi i z} \frac{\Gamma\left(\frac{p q}{t} z^{ \pm 2} ; p, q\right)}{\Gamma\left(z^{ \pm 2} ; p, q\right)} \Gamma\left(t^{1 / 2} z^{ \pm 1} z^{ \pm 1} a^{ \pm 1} ; p, q\right)\right]_{T=0}
$$

One can check expanding in $p$ and $q$ that the two expressions above agree. 


\section{The intergral equations: $A_{2}$ case}

Let us now discuss the higher rank generalization of the discussion in the previous section. Since the $A_{2}$ case is rather generic and we want to be explicit we will restrict the discussion to it. Unlike the $A_{1}$ quivers the higher rank, $A_{N-1}$, theories have many types of different punctures labeled by partitions of $N$. For example $A_{2}$ has two non-trivial types of punctures, minimal $U(1)$ puncture $(3=2+1)$ and maximal $S U(3)$ puncture $(3=1+1+1) .{ }^{9}$ The index of the $A_{2}$ theory corresponding to Riemann surface with genus $g, s_{1} S U(3)$ and $s_{2} U(1)$ punctures is assumed to be given by [3], ${ }^{10}$

$$
\mathcal{I}^{g,\left(s_{1}, s_{2}\right)}=\sum_{\lambda_{1} \geq \lambda_{2}=0}^{\infty} \mathcal{C}_{\lambda}^{2 g-2+s_{1}+s_{2}} \prod_{\ell_{1}=1}^{s_{1}} \psi_{\lambda}\left(\mathbf{z}_{\ell_{1}}\right) \prod_{\ell_{2}=1}^{s_{2}} \phi_{\lambda}\left(a_{\ell_{2}}\right),
$$

where $\lambda=\left(\lambda_{1}, \lambda_{2}\right)$. The theory corresponding to the sphere with one minimal and two maximal punctures is a free hyper-multiplet in bifundamental representation of $S U(3) \times$ $S U(3)$. The index of this theory is given by

$$
\mathcal{I}^{0,(2,1)}=\sum_{\lambda_{1} \geq \lambda_{2}=0}^{\infty} \mathcal{C}_{\lambda} \psi_{\lambda}(\mathbf{z}) \psi_{\lambda}(\mathbf{y}) \phi_{\lambda}(a)=\prod_{i, j=1}^{N} \Gamma\left(t^{\frac{1}{2}}\left(z_{i} y_{j} a\right)^{ \pm 1} ; p, q\right) .
$$

This is the $A_{2}$ generalization of (3.6). Note that we have two un-known functions, $\psi_{\mu}(\mathbf{z})$ and $\phi_{\mu}(a)$, associated to the $S U(3)$ maximal and the $U(1)$ minimal punctures respectively. The fugacities $\mathbf{z}=\left(z_{1}, z_{2}, \frac{1}{z_{1} z_{2}}\right)$ label the maximal torus of $S U(3)$ and $a$ is a $U(1)$ fugacity. The functions $\psi_{\mu}(\mathbf{z})$ are eigenfunctions of elliptic RS, but the functions $\phi_{\mu}(a)$ are not. The latter can be related to certain residues of the former $[3,29] .{ }^{11}$ The vector multiplet measure under which $\psi_{\mu}(\mathbf{z})$ are taken to be orthonormal is

$$
\left\langle\left\langle\psi_{\mu}, \psi_{\lambda}\right\rangle\right\rangle=\kappa_{2} \oint \prod_{i=1}^{2} \frac{d z_{i}}{2 \pi i z_{i}} \prod_{i \neq j} \frac{\Gamma\left(\frac{p q}{t} z_{i} / z_{j} ; p, q\right)}{\Gamma\left(z_{i} / z_{j} ; p, q\right)} \psi_{\mu}(\mathbf{z}) \psi_{\lambda}\left(\mathbf{z}^{-1}\right)=\delta_{\lambda_{1} \mu_{1}} \delta_{\lambda_{2} \mu_{2}} .
$$

In principle one can generalize the recursive procedure we described for the $A_{1}$ case to compute indices of arbitrary $A_{N-1}$ quivers. However, the new ingredient here is that not

9 The trivial partition $3=3$ corresponds to "no puncture".

10 As in the $A_{1}$ case we assume here that the flavor symmetry is large enough. E.g., for $g=1$ there is at least one minimal puncture. See $[29,8]$ for a discussion of why and when this expression for the index fails.

11 This relation won't be too useful for our perturbative technique though. 
for all types of three-punctured spheres the indices are easily computable. For instance, the class $\mathcal{S}$ theories of type $A_{2}$ have two theories corresponding to two non-trivial threepunctured spheres: one we described above (4.2), and another one with three $S U(3)$, maximal, punctures. The latter theory is an interacting strongly-coupled SCFT with no tunable couplings [30]. The index for such a theory is hard to compute in general [31]. Here knowing explicitly the functions appearing in expressions of the form (4.1) for higher rank theories gives the only known feasible tool for computing the indices. We thus turn to describe the procedure to determine these functions for the $A_{2}$ case.

From (4.2) we can get one integral equation relating the functions $\hat{\psi}_{\mu}$ and $\hat{\phi}_{\mu}$ for the $A_{2}$ case

$$
\hat{\psi}_{\lambda}(\mathbf{y}) \hat{\phi}_{\lambda}(a)=\kappa_{2} \oint \prod_{i=1}^{2} \frac{d z_{i}}{2 \pi i z_{i}} \prod_{i \neq j} \frac{\Gamma\left(\frac{p q}{t} z_{i} / z_{j} ; p, q\right)}{\Gamma\left(z_{i} / z_{j} ; p, q\right)} \prod_{i, j=1}^{3} \Gamma\left(t^{\frac{1}{2}}\left(z_{i} y_{j} a\right)^{ \pm 1} ; p, q\right) \hat{\psi}_{\lambda}\left(\mathbf{z}^{-1}\right) .
$$

The hatted eigenfunctions include the structure constants $\mathcal{C}_{\lambda}$ in them. This equation is a straightforward generalization of the $A_{1}$ equation (3.7). However, it involves now two unknown functions: one for maximal and one for minimal puncture. We need another equation to fix both functions. Such an equation is provided by Argyres-Seiberg duality [32]: two different ways to write the index of the theory associated with a sphere with two maximal and two minimal punctures [31]. We can write the index of this theory as

$$
\begin{aligned}
& \mathcal{I}^{0,(2,2)}=\sum_{\lambda_{1} \geq \lambda_{2}=0}^{\infty} \mathcal{C}_{\lambda}^{-2} \hat{\psi}_{\lambda}\left(\mathbf{y}_{\mathbf{1}}\right) \hat{\psi}_{\lambda}\left(\mathbf{y}_{\mathbf{2}}\right) \hat{\phi}_{\lambda}(a) \hat{\phi}_{\lambda}(b)= \\
& \sum_{\lambda_{1} \geq \lambda_{2}=0}^{\infty} \mathcal{C}_{\lambda}^{-2} \kappa_{1} \oint \frac{d z}{2 \pi i z} \frac{\Gamma\left(\frac{p q}{t} z^{ \pm 2} ; p, q\right)}{\Gamma\left(z^{ \pm 2} ; p, q\right)} \Gamma\left(t^{\frac{1}{2}} z^{ \pm 1} s^{ \pm 1} ; p, q\right) \hat{\psi}_{\lambda}\left(z u, z^{-1} u, u^{-2}\right) \hat{\psi}_{\lambda}\left(\mathbf{y}_{\mathbf{1}}\right) \hat{\psi}_{\lambda}\left(\mathbf{y}_{\mathbf{2}}\right)
\end{aligned}
$$

Here we have $[32,31]$

$$
s^{2}=a^{3} / b^{3}, \quad u^{2}=1 /(a b) .
$$

Using the orthogonality of $\hat{\psi}_{\lambda} \mathrm{s}$ we derive our second integral equation

$$
\hat{\phi}_{\lambda}(b) \hat{\phi}_{\lambda}(a)=\kappa_{1} \oint \frac{d z}{2 \pi i z} \frac{\Gamma\left(\frac{p q}{t} z^{ \pm 2} ; p, q\right)}{\Gamma\left(z^{ \pm 2} ; p, q\right)} \Gamma\left(t^{\frac{1}{2}} z^{ \pm 1} s^{ \pm 1} ; p, q\right) \hat{\psi}_{\lambda}\left(z u, z^{-1} u, u^{-2}\right) .
$$


Expanding as before in power series in $T$ we will get at each order a system of two linear equations,coming from (4.4) and (4.7), in two variables for the two unknown functions. The base of the recursion is again the known Schur limit $T=0$.

\subsection{Recursive solution}

The solution of the integral equations (4.4) and (4.7) when $t=q$ is known $[7,8]$

$$
\begin{aligned}
& \hat{\psi}_{\mu}^{(0)}(\mathbf{z} ; q)=\frac{1}{(1-q)^{2}\left(1-q^{2}\right)}\left(\prod_{i \neq j}^{3} \prod_{\ell=0}^{\infty} \frac{1}{1-q^{1+\ell} z_{i} / z_{j}}\right) \frac{\chi_{\mu}(\mathbf{z})}{\chi_{\mu}\left(q, q^{-1}, 1\right)}, \\
& \hat{\phi}_{\mu}^{(0)}(a ; q)=\frac{1}{(1-q)\left(1-q^{2}\right)}\left(\prod_{\ell=0}^{\infty} \frac{1}{1-q^{\frac{3}{2}+\ell} a^{ \pm 3}}\right) \frac{\chi_{\mu}\left(q^{\frac{1}{2}} a, q^{-\frac{1}{2}} a, a^{-2}\right)}{\chi_{\mu}\left(q, q^{-1}, 1\right)} .
\end{aligned}
$$

Here $\chi_{\mu}(\mathbf{z})$ are the Schur polynomials for the $A_{2}$ case. The functions $\hat{\psi}_{\lambda}^{(0)}(\mathbf{z})$ are labeled by irreps of $S U(3), \lambda=\left(\lambda_{1}, \lambda_{2}\right)$ with $\lambda_{1} \geq \lambda_{2}$, and they are orthogonal under the vector multiplet measure (4.3) when $t=q$,

$$
\kappa_{2} \prod_{i \neq j} \frac{\Gamma\left(\frac{p q}{t} z_{i} / z_{j} ; p, q\right)}{\Gamma\left(z_{i} / z_{j} ; p, q\right)} \rightarrow \Delta_{A_{2}}(\mathbf{z} ; q)=\frac{(q ; q)^{4}}{6} \prod_{i \neq j} \theta\left(q z_{i} / z_{j} ; q\right), \quad \prod_{i=1}^{3} z_{i}=1
$$

We define

$$
\begin{aligned}
& \Delta_{A_{2}}(\mathbf{z}, \mathbf{y}, a ; p, q, t)= \\
& \quad \kappa_{2} \prod_{i \neq j}^{3} \frac{\Gamma\left(\frac{p q}{t} z_{i} / z_{j} ; p, q\right)}{\Gamma\left(z_{i} / z_{j} ; p, q\right)} \prod_{i, j=1}^{3} \Gamma\left(t^{1 / 2}\left(z_{i} y_{j} a\right)^{ \pm 1} ; p, q\right)=\sum_{n=0}^{\infty} T^{n} \Delta_{A_{2}}^{(n)}(\mathbf{z}, \mathbf{y}, a ; p, q), \\
& \widetilde{\Delta}_{A_{2}}(z, a ; p, q, t)=\kappa_{1} \frac{\Gamma\left(\frac{p q}{t} z^{ \pm 2} ; p, q\right)}{\Gamma\left(z^{ \pm 2} ; p, q\right)} \Gamma\left(t^{1 / 2} z^{ \pm 1} a^{ \pm 1} ; p, q\right)=\sum_{n=0}^{\infty} T^{n} \widetilde{\Delta}_{A_{2}}^{(n)}(z, a ; p, q) .
\end{aligned}
$$

and

$$
\begin{aligned}
& \hat{\psi}_{\lambda}(z ; p, q, t)=\sum_{n=0}^{\infty} T^{n} \hat{\psi}_{\lambda}^{(n)}(\mathbf{z} ; p, q)=\sum_{n=0}^{\infty} T^{n} \sum_{\mu=0}^{\infty} C_{\mu ; \lambda}^{(n)}(p, q) \hat{\psi}_{\mu}^{(0)}(\mathbf{z} ; q), \\
& \hat{\phi}_{\lambda}(a ; p, q, t)=\sum_{n=0}^{\infty} T^{n} \hat{\phi}_{\lambda}^{(n)}(a ; p, q)=\sum_{n=0}^{\infty} T^{n} \sum_{h=-\infty}^{\infty} \widetilde{C}_{h ; \lambda}^{(n)}(p, q) a^{h} .
\end{aligned}
$$


The two integral equations of the previous sub-section become,

$$
\begin{aligned}
& \sum_{\ell=0}^{n} C_{\mu ; \lambda}^{(\ell)} \widetilde{C}_{h ; \lambda}^{(n-\ell)}=\sum_{\ell=0}^{n} \sum_{\nu} \Gamma_{\mu ; h}^{(\ell)^{\nu}} C_{\nu ; \lambda}^{(n-\ell)}, \\
& \sum_{\ell=0}^{n} \widetilde{C}_{g ; \lambda}^{(\ell)} \widetilde{C}_{h ; \lambda}^{(n-\ell)}=\sum_{\ell=0}^{n} \sum_{\nu} \widetilde{\Gamma}_{g, h}^{(\ell) \nu} C_{\nu ; \lambda}^{(n-\ell)}
\end{aligned}
$$

where

$$
\begin{aligned}
\Gamma_{h ; \nu}^{(k)^{\rho}}= & \oint \prod_{i=1}^{2} \frac{d z_{i}}{2 \pi i z_{i}} \oint \prod_{i=1}^{2} \frac{d y_{i}}{2 \pi i y_{i}} \oint \frac{d a}{2 \pi i a} \\
& \frac{\Delta_{A_{2}}^{(k)}(\mathbf{z}, \mathbf{y}, a ; q, p) \Delta_{A_{2}}(\mathbf{y} ; q) \hat{\psi}_{\rho}^{(0)}\left(\mathbf{z}^{-1} ; q\right) \hat{\psi}_{\nu}^{(0)}\left(\mathbf{y}^{-1} ; q\right) a^{-h}}{\left\langle\hat{\psi}_{\nu}^{(0)}, \hat{\psi}_{\nu}^{(0)}\right\rangle}, \\
\widetilde{\Gamma}_{g, h}^{(k) \rho}= & \oint \frac{d z}{2 \pi i z} \oint \frac{d a}{2 \pi i a} \oint \frac{d b}{2 \pi i b} \widetilde{\Delta}_{A_{2}}^{(k)}\left(z,\left(\frac{a}{b}\right)^{\frac{3}{2}} ; q, p\right) \hat{\psi}_{\rho}^{(0)}\left(\frac{z}{\sqrt{a b}}, \frac{z^{-1}}{\sqrt{a b}}, a b ; q\right) a^{-g} b^{-h} .
\end{aligned}
$$

At the base of the recursion, $n=0$, we have

$$
\delta_{\mu \lambda} \widetilde{C}_{h ; \lambda}^{(0)}=\Gamma_{\mu ; h}^{(0)^{\lambda}}, \quad \widetilde{C}_{g ; \lambda}^{(0)} \widetilde{C}_{h ; \lambda}^{(0)}=\widetilde{\Gamma}_{g, h}^{(0) \lambda} .
$$

Let us derive the leading correction to the $A_{2}$ eigenfunctions. From the first equation in (4.12) we get

$$
C_{\mu ; \lambda}^{(1)}\left(\widetilde{C}_{h ; \lambda}^{(0)}-\widetilde{C}_{h ; \mu}^{(0)}\right)+\delta_{\mu \lambda} \widetilde{C}_{h ; \lambda}^{(1)}=\Gamma_{\mu ; h}^{(1)^{\lambda}}
$$

and in particular,

$$
\begin{array}{ll}
\mu \neq \lambda: & C_{\mu ; \lambda}^{(1)}\left(\widetilde{C}_{h ; \lambda}^{(0)}-\widetilde{C}_{h ; \mu}^{(0)}\right)=\Gamma_{\mu ; h}^{(1)^{\lambda}}, \\
\mu=\lambda: & \widetilde{C}_{h ; \lambda}^{(1)}=\Gamma_{\lambda ; h}^{(1)^{\lambda} .}
\end{array}
$$

Thus we completely fix $\widetilde{C}_{h ; \lambda}^{(1)}$ and $C_{\mu ; \lambda}^{(1)}$ for $\mu \neq \lambda$. To solve for $C_{\lambda ; \lambda}^{(1)}$ one needs the second equation in (4.12). Note also that in the first line in (4.16) the value of $h$ is arbitrary and thus this equation encodes a large number of consistency conditions. The second equation in (4.12) for $n=1$ gives 


$$
\widetilde{C}_{g ; \lambda}^{(1)} \widetilde{C}_{h ; \lambda}^{(0)}+\widetilde{C}_{g ; \lambda}^{(0)} \widetilde{C}_{h ; \lambda}^{(1)}=\widetilde{\Gamma}_{g, h}^{(1) \lambda}+\sum_{\nu} \widetilde{\Gamma}_{g, h}^{(0) \nu} C_{\nu ; \lambda}^{(1)}
$$

From here we deduce

$$
C_{\lambda ; \lambda}^{(1)} \widetilde{\Gamma}_{g, h}^{(0) \lambda}=\Gamma_{\lambda ; g}^{(1)^{\lambda}} \widetilde{C}_{h ; \lambda}^{(0)}+\Gamma_{\lambda ; h}^{(1)^{\lambda}} \widetilde{C}_{g ; \lambda}^{(0)}-\widetilde{\Gamma}_{g, h}^{(1) \lambda}-\sum_{\nu \neq \lambda} \widetilde{\Gamma}_{g, h}^{(0) \nu} \frac{\Gamma^{(1)}{ }_{\nu ; \hat{h}}^{\lambda}}{\widetilde{C}_{\hat{h} ; \lambda}^{(0)}-\widetilde{C}_{\hat{h} ; \nu}^{(0)}}
$$

where $\hat{h}$ is any integer such that $\widetilde{C}_{\hat{h} ; \lambda}^{(0)}-\widetilde{C}_{\hat{h} ; \nu}^{(0)} \neq 0$. This completes the determination of the leading corrections and gives yet another set of consistency equations. For general order in $T$ expansion the first equation in (4.12) with $\mu \neq \lambda$ detemines $C_{\mu ; \lambda}^{(n)}$ and with $\mu=\lambda$ it determines $\widetilde{C}_{h ; \lambda}^{(n)}$, while the second equation in (4.12) determines $C_{\lambda ; \lambda}^{(n)}$.

One can make several consistency checks of the procedure. Let us mention a simple non-trivial example of such a check. The flavor symmetry of $\mathcal{I}^{0,(3,0)}$ is $S U(3)^{3}$ but is actually supposed to enhance to $E_{6}$ [1]. In particular, the symmetry of $\mathcal{I}^{1,(1,0)}$ is $G_{2}$ [29]: we can check that this enhancement of the flavor symmetry indeed occurs for the solution of our perturbative algorithm. The index $\mathcal{I}_{1}^{1,(1,0)}$ is given by

$$
\begin{aligned}
\mathcal{I}_{1}^{(1,(1,0))}(\mathbf{z})= & \sum_{\lambda} \hat{\psi}_{\lambda}^{(1)}(\mathbf{z})=\sum_{\lambda}\left\{\left(\frac{\Gamma_{\lambda ; g}^{(1)^{\lambda}}}{\widetilde{C}_{g ; \lambda}^{(0)}}+\frac{\Gamma_{\lambda ; h}^{(1)}{ }_{\lambda ; h}^{\lambda}}{\widetilde{C}_{h ; \lambda}^{(0)}}-\frac{\widetilde{\Gamma}_{g, h}^{(1) \lambda}}{\widetilde{C}_{g ; \lambda}^{(0)} \widetilde{C}_{h ; \lambda}^{(0)}}\right) \hat{\psi}_{\lambda}^{(0)}(\mathbf{z})+\right. \\
& \left.\sum_{\nu \neq \lambda}\left(\left[\hat{\psi}_{\nu}^{(0)}(\mathbf{z})-\hat{\psi}_{\lambda}^{(0)}(\mathbf{z}) \frac{\widetilde{C}_{g ; \nu}^{(0)} \widetilde{C}_{h ; \nu}^{(0)}}{\widetilde{C}_{g ; \lambda}^{(0)} \widetilde{C}_{h ; \lambda}^{(0)}}\right] \frac{\Gamma_{\nu ; \hat{h}}^{(1)}{ }_{\nu}^{\lambda}}{\widetilde{C}_{\hat{h} ; \lambda}^{(0)}-\widetilde{C}_{\hat{h} ; \nu}^{(0)}}\right)\right\} .
\end{aligned}
$$

The fugacities $z_{1}$ and $z_{2}$ label the Cartans of $S U(3)$ but actually the symmetry here is enhanced to $G_{2}$ and this index has a natural expansion in terms of $G_{2}$ characters labeled by the same parameters. To the lowest orders we have

$$
\mathcal{I}_{1}^{(1,(1,0))}(\mathbf{z})=2 q(1+p) \chi_{14}^{G_{2}}(\mathbf{z})-2 p q \chi_{7}^{G_{2}}(\mathbf{z})+\cdots
$$

This expression can be checked against the results of [31] for the index $\mathcal{I}^{0,(3,0)}$ obtained by using an inversion formula [33] of the integral equation at hand (4.7).

Yet another, simpler, consistency check of our procedure is the computation of $\mathcal{I}_{1}^{1,(0,1)}$ which is given by 


$$
\mathcal{I}_{1}^{(1,(0,1))}(a)=\sum_{\lambda} \hat{\phi}_{\lambda}^{(1)}(a)=\sum_{\lambda} \sum_{h=-\infty}^{\infty} \Gamma_{\lambda ; h}^{(1)^{\lambda}} a^{h},
$$

and can be explcitly seen to match, expanding in $p$ and $q$, the same index computed by gauging a diagonal combination of two $S U(3)$ symmetries in $\mathcal{I}^{0,(2,1)}$ given in (4.2).

\section{Comments}

Let us make several brief remarks about our results and further research directions.

1 . Assuming S-duality and making certain mathematical assumptions about the structure of the index of theories of class $\mathcal{S}$ we derived a simple perturbative algorithm to compute the index. This algorithm involves determining a set of eigenfunctions of the elliptic RS model. We gave explicit details of the $A_{1}$ and the $A_{2}$ cases. The knowledge of the eigenfunctions up to a finite order in the expansion parameter is sufficient to compute exactly a (finite) set of indices generalizing the Schur index.

2 . The system of recursive equations determining the eigenfunctions needed to compute the index is highly overdetermined. We have checked that our procedure is consistent to lowest orders in the expansion parameter. It would be very beneficial to verify/prove whether the procedure makes sense to all orders. In particular it should be enlightening in many respects to obtain closed form expressions for the relevant eigenfunctions.

3 . The algorithm discussed in this note, although stated for $A_{1}$ and $A_{2}$ cases, can be generalized in principle to higher rank theories. There we have more types of punctures with a priori un-known functions associated to each one of them. But we also have more non-trivial dualities [1] generalizing the Argyres-Seiberg duality which can be translated into integral equations relating the different un-known functions. It should be interesting to study the general structure of these systems of integral equations.

4. As a base of our recursive procedure we have used the known expressions for the index in the Schur case, $t=q$. One can try and use the known solution of the integral equations in the Macdonald case, $p=0$, as a starting point for the perturbative construction of the eigenfunctions. 


\section{Acknowledgments:}

We would like to thank A. Gadde, D. Gaiotto, E. Langmann, L. Rastelli, Y. Tachikawa, and B. Willett for very interesting discussions. We are grateful to the organizers of the workshop "Elliptic Integrable Systems and Hypergeometric Functions ", (July 2013, Lorentz Center), the organizers of the Simons Summer Workshop in Stony Brook (August 2013), and to the high energy theory group at the Weizmann institute for hospitality during different stages of this project.We gratefully acknowledge support from the Martin A. Chooljian and Helen Chooljian membership at the Institute for Advanced Study. This research was also partially supported by NSF grant number PHY-0969448. 


\section{References}

[1] D. Gaiotto, "N=2 dualities," JHEP 1208, 034 (2012). [arXiv:0904.2715 [hep-th]].

[2] D. Gaiotto, G. W. Moore and A. Neitzke, "Wall-crossing, Hitchin Systems, and the WKB Approximation," [arXiv:0907.3987 [hep-th]].

[3] D. Gaiotto, L. Rastelli and S. S. Razamat, "Bootstrapping the superconformal index with surface defects," JHEP 1301, 022 (2013). [arXiv:1207.3577 [hep-th]].

[4] S. N. M. Ruijsenaars and H. Schneider, "A New Class Of Integrable Systems And Its Relation To Solitons," Annals Phys. 170, 370 (1986).

[5] S. N. M. Ruijsenaars, "Complete Integrability Of Relativistic Calogero-moser Systems And Elliptic Function Identities," Commun. Math. Phys. 110, 191 (1987).

[6] Y. Komori and S. Ruijsenaars, "Elliptic integrable systems of Calogero-Moser type: A survey", in Proceedings of the 2004 Kyoto Workshop on "Elliptic integrable systems", (M. Noumi, K. Takasaki, Eds.), Rokko Lectures in Math., no. 18, Dept. of Math., Kobe Univ.

[7] A. Gadde, L. Rastelli, S. S. Razamat and W. Yan, "The 4d Superconformal Index from q-deformed 2d Yang-Mills," Phys. Rev. Lett. 106, 241602 (2011). [arXiv:1104.3850 [hep-th]].

[8] A. Gadde, L. Rastelli, S. S. Razamat and W. Yan, "Gauge Theories and Macdonald Polynomials," Commun. Math. Phys. 319, 147 (2013). [arXiv:1110.3740 [hep-th]].

[9] Y. Tachikawa, "4d partition function on $S^{1} \times S^{3}$ and 2d Yang-Mills with nonzero area," PTEP 2013, $013 B 01$ (2013). [arXiv:1207.3497 [hep-th]].

[10] Y. Fukuda, T. Kawano and N. Matsumiya, "5D SYM and 2D q-Deformed YM," Nucl. Phys. B 869, 493 (2013). [arXiv:1210.2855 [hep-th]].

[11] L. F. Alday, M. Bullimore, M. Fluder and L. Hollands, "Surface defects, the superconformal index and q-deformed Yang-Mills," [arXiv:1303.4460 [hep-th]].

[12] S. S. Razamat, "On a modular property of $\mathrm{N}=2$ superconformal theories in four dimensions," JHEP 1210, 191 (2012). [arXiv:1208.5056 [hep-th]].

[13] J. Kinney, J. M. Maldacena, S. Minwalla and S. Raju, "An Index for 4 dimensional super conformal theories," Commun. Math. Phys. 275, 209 (2007). [hep-th/0510251].

[14] A. Gorsky and N. Nekrasov, "Relativistic Calogero-Moser model as gauged WZW theory," Nucl. Phys. B 436, 582 (1995). [hep-th/9401017].

[15] A. Gorsky and N. Nekrasov, "Elliptic Calogero-Moser system from two-dimensional current algebra," [hep-th/9401021].

[16] N. Nekrasov, "Five dimensional gauge theories and relativistic integrable systems," Nucl. Phys. B 531, 323 (1998). [hep-th/9609219].

[17] A. Gorsky and A. Mironov, "Integrable many body systems and gauge theories," In *Aratyn, H. (ed.) et al.: Integrable hierarchies and modern physical theories* 33-176. [hep-th/0011197]. 
[18] N. A. Nekrasov and S. L. Shatashvili, "Quantization of Integrable Systems and Four Dimensional Gauge Theories," [arXiv:0908.4052 [hep-th]].

[19] D. Gaiotto and P. Koroteev, "On Three Dimensional Quiver Gauge Theories and Integrability," JHEP 1305, 126 (2013). [arXiv:1304.0779 [hep-th]].

[20] A. Gadde, E. Pomoni, L. Rastelli and S. S. Razamat, "S-duality and 2d Topological QFT," JHEP 1003, 032 (2010). [arXiv:0910.2225 [hep-th]].

[21] D. Xie, "General Argyres-Douglas Theory," JHEP 1301, 100 (2013). [arXiv:1204.2270 [hep-th]].

[22] F. J. van de Bult, "An elliptic hypergeometric integral with $W\left(F_{4}\right)$ symmetry," The Ramanujan Journal, Volume 25, Issue 1 (2011) [arXiv:0909.4793[math.CA]].

[23] M. Rahman, A. Verma, "Product and Addition Formulas for the Continuous qUltraspherical Polynomials," SIAM J. Math. Anal., 17(6).

[24] E. Langmann, "Explicit solution of the (quantum) elliptic Calogero-Sutherland model", [arXiv:math-ph/0401029].

[25] S. Ruijsenaars, "Elliptic integrable systems of Calogero-Moser type: Some new results on joint eigenfunctions", in Proceedings of the 2004 Kyoto Workshop on "Elliptic integrable systems", (M. Noumi, K. Takasaki, Eds.), Rokko Lectures in Math., no. 18, Dept. of Math., Kobe Univ.

[26] M. Hallnas and S. Ruijsenaars, "Kernel functions and Baecklund transformations for relativistic Calogero-Moser and Toda systems," J. Math. Phys. 53, 123512 (2012).

[27] Y. Komori, M. Noumi, J. Shiraishi, "Kernel Functions for Difference Operators of Ruijsenaars Type and Their Applications," SIGMA 5 (2009), 054. [arXiv:0812.0279 [math.QA]].

[28] E. Langmann, "Conformal field theory and the solution of the (quantum) elliptic Calogero-Sutherland system", [arXiv:math-ph/0411081].

[29] D. Gaiotto and S. S. Razamat, "Exceptional Indices," JHEP 1205, 145 (2012). [arXiv:1203.5517 [hep-th]].

[30] J. A. Minahan and D. Nemeschansky, "An N=2 superconformal fixed point with $\mathrm{E}(6)$ global symmetry," Nucl. Phys. B 482, 142 (1996). [hep-th/9608047].

[31] A. Gadde, L. Rastelli, S. S. Razamat and W. Yan, "The Superconformal Index of the $E_{6}$ SCFT," JHEP 1008, 107 (2010). [arXiv:1003.4244 [hep-th]].

[32] P. C. Argyres and N. Seiberg, "S-duality in N=2 supersymmetric gauge theories," JHEP 0712, 088 (2007). [arXiv:0711.0054 [hep-th]].

[33] V. P. Spiridonov and S. O. Warnaar, "Inversions of integral operators and elliptic beta integrals on root systems," Adv. Math. 207 (2006), 91-132 [arXiv:math/0411044]. 